\title{
PTEN regulates plasma membrane expression of glucose transporter 1 and glucose uptake in thyroid cancer cells
}

Federica Morani', Suratchanee Phadngam', Carlo Follo', Rossella Titone', Gianluca Aimaretti ${ }^{2}$, Alessandra Galetto ${ }^{3}$, Oscar Alabiso ${ }^{3}$ and Ciro Isidoro'

${ }^{1}$ Laboratory of Molecular Pathology and Nanobioimaging, Department of Health Sciences, ${ }^{2}$ Unit of Clinical Endocrinology, and ${ }^{3}$ Unit of Oncology, Department of Translational Medicine, Università del Piemonte Orientale 'A. Avogadro', Via Solaroli 17, 28100 Novara, Italy
Correspondence should be addressed to $\mathrm{C}$ Isidoro

Email

isidoro@med.unipmn.it

\begin{abstract}
Glucose represents an important source of energy for the cells. Proliferating cancer cells consume elevated quantity of glucose, which is converted into lactate regardless of the presence of oxygen. This phenomenon, known as the Warburg effect, has been proven to be useful for imaging metabolically active tumours in cancer patients by ${ }^{18} \mathrm{~F}$-fluorodeoxyglucose positron emission tomography (FDG-PET). Glucose is internalised in the cells by glucose transporters (GLUTs) belonging to the GLUT family. GLUT1 (SLC2A1) is the most prevalent isoform in more aggressive and less differentiated thyroid cancer histotypes. In a previous work, we found that loss of expression of PTEN was associated with increased expression of GLUT1 on the plasma membrane (PM) and probability of detecting thyroid incidentalomas by FDG-PET. Herein, we investigated the molecular pathways that govern the expression of GLUT1 on the PM and the glucose uptake in WRO (expressing WT PTEN) and FTC133 (PTEN null) follicular thyroid cancer cells cultured under glucose-depleted conditions. The membrane expression of GLUT1 was enhanced in glucose-deprived cells. Through genetic manipulations of PTEN expression, we could demonstrate that the lack of this oncosuppressor has a dominant effect on the membrane expression of GLUT1 and glucose uptake. We conclude that loss of function of PTEN increases the probability of cancer detection by FDG-PET or other glucose-based imaging diagnosis.
\end{abstract}
Key Words
- PTEN
- glucose
- Warburg effect
- FDG-PET imaging, cancer
- GLUT

Journal of Molecular Endocrinology (2014) 53, 247-258

\section{Introduction}

Glucose is a fundamental source of energy for mammalian cells. Normally, in the presence of oxygen, glucose is oxidised through the mitochondrial respiration pathway with the highest rate of production of ATP. However, in intensely proliferating cancer cells, glucose is preferentially converted into lactate despite the presence of oxygen and functional mitochondria. This aberrant metabolism of glucose, known as the Warburg effect or aerobic glycolysis, is much less convenient in terms of energy gain and imposes a large consumption of glucose in proliferating cells (Newsholme et al. 1985, Koppenol et al. 2011). This fact is being exploited for diagnostic imaging of tumours in patients injected with the probe ${ }^{18} \mathrm{~F}$-fluorodeoxyglucose (FDG), a radioactive analogue of glucose,

Published by Bioscientifica Ltd. 
which can be detected by positron emission tomography (PET) (Busk et al. 2008). As the uptake of FDG competes with that of circulating glucose, the patient undergoing FDG-PET must avoid the intake of any source of glucose during the $6 \mathrm{~h}$ preceding the start of the PET study (Boellaard et al. 2010). This technique has the potential to reveal unsuspected tumours, commonly indicated as 'incidentaloma' because of their accidental discovery.

Glucose, similar to its radioactive analogue ${ }^{18} \mathrm{FDG}$ and its fluorescent equivalent 2-( $N$-(7-nitrobenz2-oxa-1,3-diazol-4-yl)amino)-2-deoxyglucose (2-NBDG), is internalised in the cells by glucose transporters (GLUTs) belonging to the GLUT family (Szablewski 2013). GLUT1 (SLC2A1) is the most prevalent isoform in highly aggressive and less differentiated thyroid cancer histotypes (Hosaka et al. 1992, Samih et al. 2000, Matsuzu et al. 2005).

Only a few studies have addressed the signalling pathways involved in the glucose uptake by GLUT1 in thyroid cancer cells. There are indications that the surface expression of GLUT proteins is under the control of the phosphoinositide 3-kinase (PI3K)/AKT pathway (Samih et al. 2000). Recently, AMPK has been shown to play a pivotal role in the membrane expression of GLUT1 and glucose uptake in rat follicular thyroid cells (Andrade et al. 2012). In a small cohort of patients, we found that loss of expression of the oncosuppressor PTEN, a protein-lipid phosphatase that switches off the AKT pathway, was associated with an increased probability of detecting thyroid incidentalomas by FDG-PET (Morani et al. 2012). In this work, we analysed the signalling pathways that govern the expression of GLUT1 on the plasma membrane (PM; and the uptake of glucose) in two follicular thyroid cancer cell lines: WRO, expressing WT PTEN, and FTC133 PTEN null cells. Genetic manipulations indicated that the lack of PTEN expression has a dominant effect on the membrane expression of GLUT1 and glucose uptake. This finding substantiates our hypothesis that loss of function of PTEN increases the probability of cancer detection by FDG-PET or other glucose-based imaging diagnosis (Morani et al. 2012).

\section{Materials and methods}

\section{Thyroid cancer cell lines, cell culture conditions and pharmacological treatments}

The WRO and FTC133 follicular thyroid cancer cell lines were kindly provided by Dr Francesco Frasca, University of Catania, Italy.
The WRO cell line was cultured in RPMI-1640 media (with L-glutamine) supplemented with foetal bovine serum (FBS, 10\%) and penicillin/streptomycin (1\%); the FTC133 cell lines were cultured in DMEM, nutrient mixture F-12 (1:1, by volume), supplemented with FBS (10\%), penicillin/streptomycin (1\%) and L-glutamine (1\%). All culture reagents were purchased from SigmaAldrich. For studies on glucose deprivation, the cells were incubated in a glucose-free medium (R1383 and D5030, Sigma-Aldrich). For the experiments, growing cells were plated on sterile plastic dishes and allowed to adhere for at least $24 \mathrm{~h}$ before use. The PI3K inhibitor 3-methyladenine (3MA; Sigma-Aldrich) was used at a concentration of $10 \mathrm{mM}$. At the end of the incubations, media and cells or coverslips were collected and processed for protein analysis as detailed below.

\section{siRNA silencing of PTEN}

Post-transcriptional silencing was achieved by the siRNA technology. Duplexes of nucleotide siRNA were synthesised by MWG Biotech AG (Ebersberg, Germany). The sequence and use of the siRNA for Sham transfection have been described previously (Trincheri et al. 2007). Two siRNA oligos targeting two different exons were used for the silencing of PTEN, and the sense strands were $5^{\prime}$-AGACUUGAAGGCGUAUACA-3' and 5'-AGAAUCAUCUGGAUUAUAG- $3^{\prime}$. Adherent cells (plated at a density of $30000 / \mathrm{cm}^{2}$ in a Petri dish) were incubated for $6 \mathrm{~h}$ with 100 pmol RNA duplexes in the presence of $6 \mu$ l Lipofectamine 2000 (Invitrogen Co.) in $500 \mu \mathrm{l}$ Optimem (Life Technologies Co.). The cells were then washed and treated $36 \mathrm{~h}$ after transfection to allow for a maximal effect on protein down-regulation.

\section{Plasmid transfections}

pcDNA3.1Zeo + plasmid containing the full-length WT PTEN cDNA were transfected by Lipofectamine 2000 (Invitrogen Co.) following the manufacturer's protocol. The full-length PTEN cDNA was cloned from OAW42 ovarian cancer cells mRNA and fully sequenced to confirm the WT sequence. OAW42 total RNA was extracted according to the TRIzol LS reagent protocol (Life Technologies Co.). Total RNA ( $3 \mu \mathrm{g})$ was retrotranscribed using the RevertAiD H Minus FirstStrand cDNA Synthesis Kit (Fermentas, Burlington, CA, USA). RT-PCR was performed according to the manufacturer's instructions with DyNzyme EXT DNA Polymerase (Finnzymes OY, Espoo, Finland) starting from $2 \mu \mathrm{l}$ cDNA and using a final

Published by Bioscientifica Ltd 
concentration of $10 \mu \mathrm{M}$ PTEN primers (forward primer, CATTTCCATCCTGCAGAAGAAG and reverse primer, CCCAATACAGATTCACTTCCTTTAG). PTEN cDNA was subcloned into the plasmid pcDNA 3.1 Zeo (Life Technologies Co.) and subjected to automated sequencing (ABI PRISM 3100, Applied Biosystems). Primers were obtained from MWG-BIOTECH AG (Ebersberg, Germany). An empty pcDNA3.1Zeo + plasmid was used as control transfection (Sham).

\section{Glucose uptake assay}

The fluorescent glucose analogue 2-NBDG (Life Technologies Co.) was used to detect glucose uptake in living cells (O'Neil et al. 2005). Cells grown on coverslips were incubated with $50 \mu \mathrm{M}$ of 2 -NBDG for $1 \mathrm{~h}$ before the end of the treatments, washed twice with PBS $1 \times$ and rapidly imaged under a fluorescence microscope (Leica DMI6000B, Leica Microsystem AG, Wetzlad, Germany).

\section{Immunofluorescence staining}

At the end of treatments, cells adherent on sterile glass coverslips were fixed in cold methanol for $20 \mathrm{~min}$ and permeabilised with $0.2 \%$ Triton $\mathrm{X}-100$ in PBS $1 \times$ for $10 \mathrm{~min}$. Antigens were revealed with a primary specific antibody followed by IRIS2 (green fluorescence)-conjugated goat anti-rabbit IgG or IRIS3 (red fluorescence)conjugated goat anti-mouse IgG (Cyanine Technologies SpA, Turin, Italy) secondary antibody, as appropriate, for $1 \mathrm{~h}$ at room temperature in a humid chamber. The nucleus was evidenced by staining the chromatin with the fluorescent dye 4,6-diamidino-2-phenylindole-dihydrochloride (DAPI). As negative control, the primary antibody was omitted or substituted with pre-immune antiserum. Antibodies were diluted in PBS containing 0.1\% Triton X-100 and 10\% FBS. The following primary antibodies were used: rabbit polyclonal anti-GLUT1 (Millipore, Darmstadt, Germany), rabbit polyclonal anti-PTEN (Millipore) and mouse monoclonal anti-Rab5 (BD Transduction Laboratories, San Jose, CA, USA). Stained cells were mounted with Slow-FADE (Light AntiFADE Kit, Molecular Probes Invitrogen) and observed under a fluorescence microscope (Leica DMI6000B, Leica Microsystem AG). Observations were performed by two independent investigators. Representative images of at least three independent experiments are shown. Image processing was carried out using the ImageJ Software (freely available at http://imagej.nih.gov/ij/, see below).

\section{Western blotting assay}

Immunoblotting was carried out following standard procedures as reported previously (Castino et al. 2007). Cell homogenates were prepared by freeze-thawing and

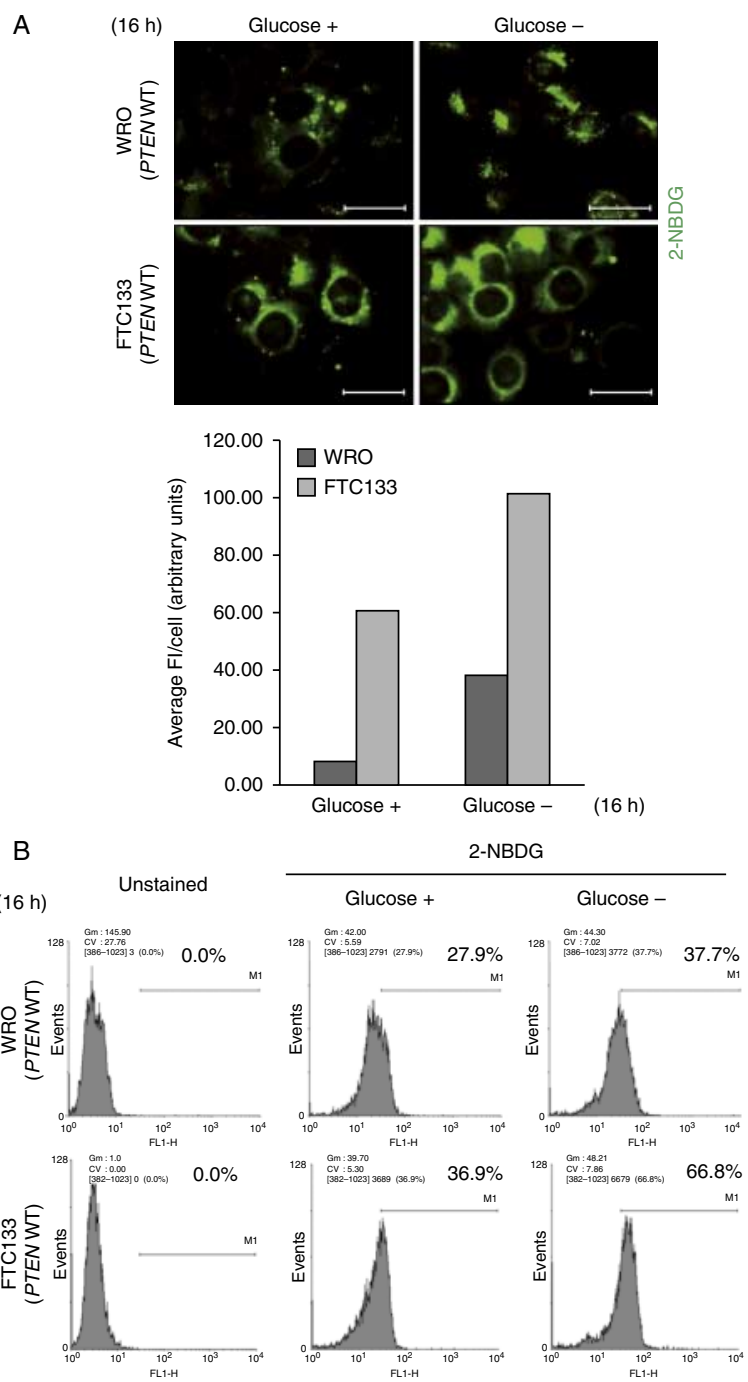

\section{Figure 1}

Differential uptake of glucose in WRO and FTC133 cells. (A) The uptake of the glucose fluorescent analogue 2-NBDG (in green, $50 \mu \mathrm{M}$ ) was monitored for $16 \mathrm{~h}$ in both cell lines cultured in a glucose-containing or glucose-free medium. The probe was added to the culture medium $1 \mathrm{~h}$ before the end of the incubation. Representative microscopic fields are shown in the upper panel. Quantification of 2-NBDG uptake was done using the ImageJ Software directly on the microscopic images of labelled cells (lower panel). (B) A parallel culture of cells plated on Petri dishes and labelled as above was used for cytofluorometric quantification of 2-NBDG-positive cells. The level of 2-NBDG uptake was higher in FTC133 cells than in WRO cells and greatly increased when the cells were pre-incubated in a glucose-free medium. Representative data of four experiments are shown. Scale bar $=20 \mu \mathrm{m}$. Magnification $=63 \times$. Data shown in this figure have been reproduced six times independently. Full colour version of this figure available via http://dx.doi.org/10.1530/JME-14-0118.

Published by Bioscientifica Ltd. 
ultrasonication in a buffer containing detergents and protease inhibitors. Approximately $30 \mu \mathrm{g}$ of cell proteins were denatured with Laemmli sample buffer, separated by electrophoresis on a $12.5 \%$ SDS-containing polyacrylamide gel and then electroblotted onto a PVDF membrane (Carlo Erba Reagents, Milan, Italy).

Protein of interest was detected with the following primary antibodies: rabbit polyclonal anti-PTEN (EX-BIO, Vestec, Czech Republic); rabbit polyclonal anti-phospho AKT (Ser473) (Cell Signaling Technology, Danvers, MA, USA); rabbit polyclonal anti-AKT (Cell Signaling Technology); rabbit polyclonal anti-GLUT1 (Millipore) and rabbit polyclonal anti-HIF1 $\alpha$ (GeneTex, Irvine, CA, USA). As an index of homogenate protein loading in the lanes, $\beta$-tubulin was used (Sigma-Aldrich). Immunocomplexes were revealed using a peroxidase-conjugated secondary antibody (Bio-Rad), as appropriate, and subsequent peroxidase-induced chemiluminescence reaction (PerkinElmer, Waltham, MA, USA). Western blotting data were reproduced at least three times independently.
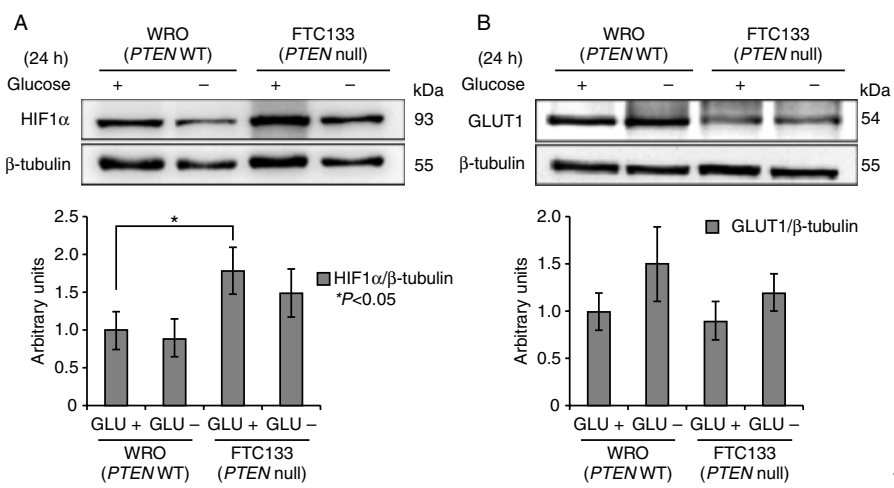

Figure 2

Differential subcellular localisation of GLUT1 in WRO and FTC133 cell lines. (A) Western blotting of HIF1 $\alpha$ in homogenates from WRO (PTEN WT) and FTC133 (PTEN null) thyroid cancer cell lines incubated for $24 \mathrm{~h}$ in a glucosecontaining or glucose-free medium. Densitometry of three experiments is included. The basal level of HIF1 $\alpha$ expression was higher in FTC133 cells than in WRO cells. (B) Western blotting of GLUT1 in homogenates prepared as for A. Densitometry of three experiments is included. Differences in the expression of GLUT1 in WRO and FTC133 cells were not statistically significant. Glucose starvation apparently did not alter the levels of HIF1 $\alpha$ and GLUT1 expression in both cell lines. (C) Immunofluorescence assay of GLUT1 expression in WRO and FTC133 cells cultured for 1 or $16 \mathrm{~h}$ in a glucosecontaining or glucose-free medium. Representative fields of immunofluorescence staining are shown in the upper panels, and the corresponding

\section{Quantification analysis: methods and statistics}

Unless otherwise specified, all experiments were replicated three times independently and in duplicate or triplicate. For measurement of glucose uptake, two methods were employed. In adherent 2-NBDG-loaded cells, data quantification of the area and of the intensity of fluorescence images was performed using the Image $1.48 \mathrm{v}$ Software (freely available at http://imagej.nih.gov/ij/). At least five randomly chosen fields for a total of minimum 50 cells were analysed. Fluorescence intensity (FI) is given in arbitrary units as an average value per cell in the selected representative fields.

Alternatively, the 2-NBDG-loaded cells were subjected to cytofluorometry analysis. Adherent cells were incubated with $50 \mu \mathrm{M}$ of 2 -NBDG for $1 \mathrm{~h}$ before the end of the treatments, washed twice with PBS $1 \times$, collected by trypsinisation, centrifuged at $100 \mathrm{~g}$ at $4{ }^{\circ} \mathrm{C}$ for $10 \mathrm{~min}$ and re-suspended in OPTIMEM (Life Technologies Co.). Cells were analysed using a Becton Dickinson FACS SCAN cytofluorometer (BD Bioscience, Franklin Lakes, NJ, USA).
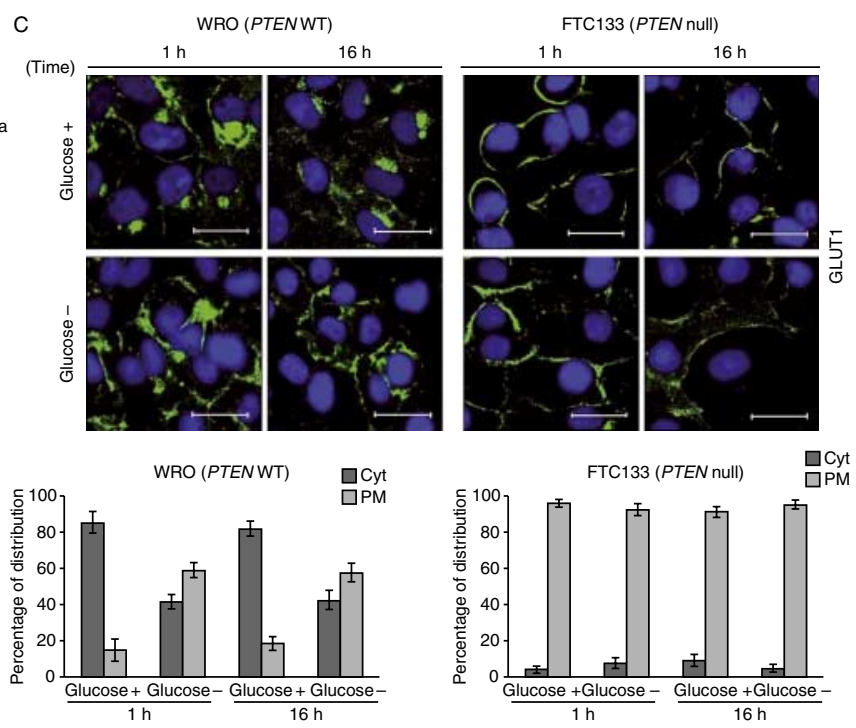

fluorescence quantification of GLUT1 in the cytoplasmic (Cyt) and plasma membrane (PM) compartments is shown in the lower panels. In WRO cells cultured in a glucose-containing medium, GLUT1 was localised to the cytoplasm ( $\sim 85 \%)$ and, under glucose-depleted conditions, it translocated onto the PM (from $\sim 15$ to $\sim 60 \%$ ). In FTC133 cells, GLUT1 permanently localised to the PM ( $95 \%)$, regardless of the presence or absence of glucose in the culture medium. Nuclei are stained with DAPI. The images shown in $\mathrm{C}$ have been reproduced four times independently. Fluorescence quantification is based on the analysis of a minimum of 50 cells for each of the three independent experiments. Scale bar $=20 \mu \mathrm{m}$. Magnification $=63 \times$. Full colour version of this figure available via http://dx.doi.org/10.1530/JME-14-0118.

Published by Bioscientifica Ltd. 
Data were acquired using the CellQuest Software (BD Bioscience) (10 000 gated events/sample, on FL-1) and analysed using the WinMDI 2.9 Software (http://facs. scripps.edu/software.htm). Unstained parallel cultures were used for setting the instrument. The proportion of cells positive for 2-NBDG uptake is given as the percentage of the whole population. One (out of three or four) representative cytofluorogram is shown per condition. Alternatively, data are expressed as average \pm s.D. of $n=4$.

Quantification of protein expression in fluorescence images of adherent cells was performed using the ImageJ Software. Data were collected from at least three independent experiments. Five randomly chosen fields for a total of minimum 50 cells were analysed in each coverslip. Data on subcellular distribution of the protein of interest (GLUT1) are expressed as percentage ( \pm s.D.) of FI in the PM and cytoplasmic (Cyt) compartments of the cells in the selected representative fields.
Densitometry quantification of western blotting bands was performed using both the ImageJ (1.48v; http://imagej.nih.gov/ij/) and Quantity One 4.5.0 Softwares (Bio-Rad), with consistent results. Data are expressed in arbitrary units as average \pm s.D. of at least three independent experiments. The $P$ values $<0.05$ were considered statistically significant.

\section{Results}

FTC133 cells take up glucose more avidly than WRO cells

To monitor the ability of cells to take up glucose, we employed 2-NBDG, a glucose analogue fluorescently labelled at position 2, that is a substrate for GLUTs (Fig. 1). Quantification of 2-NBDG uptake was performed measuring the fluorescence in the cell monolayer (Fig. 1A, lower panel) as well as by cytofluorometry after
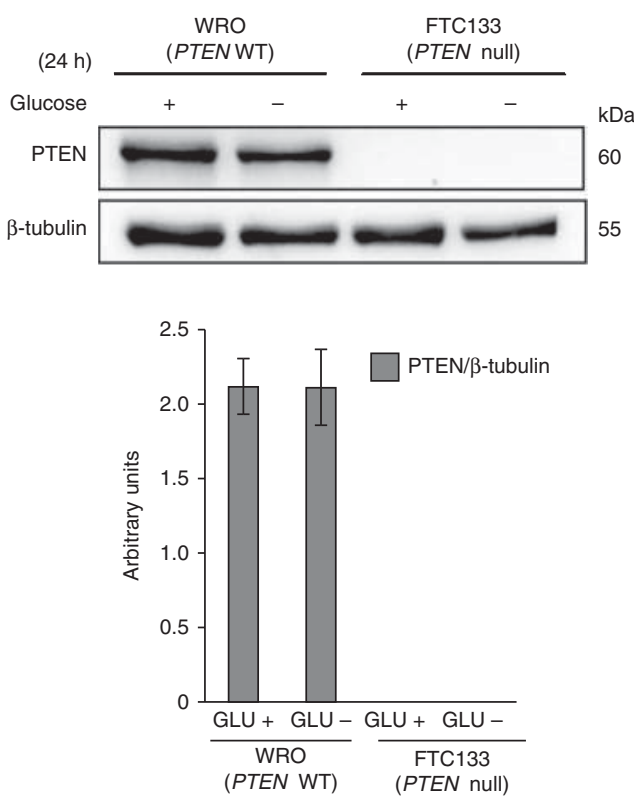
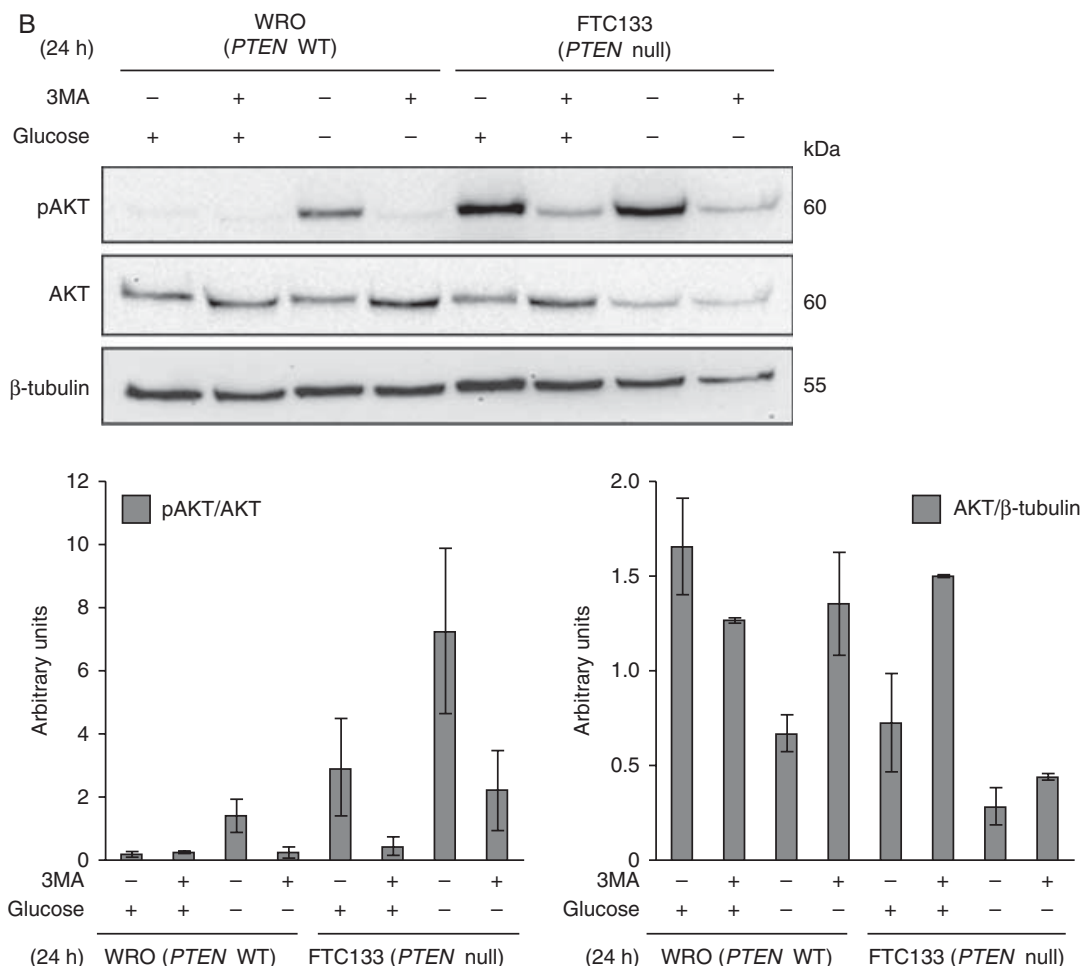

\section{Figure 3}

Involvement of the PI3K signalling pathway in the glucose-deprivation response in WRO and FTC133 cells. (A) PTEN status was analysed by western blotting in WRO and FTC133 cell lines after $24 \mathrm{~h}$ of incubation in a glucosecontaining and glucose-free media. FTC133 cells do not express detectable levels of PTEN. In WRO cells, the expression of PTEN is not subjected to glucose-dependent modulation. (B) AKT status, as a readout of PI3K activity, was analysed by western blotting in WRO and FTC133 cells cultured
C 2014 Society for Endocrinology Printed in Great Britain for $24 \mathrm{~h}$ in the presence or absence of glucose and of the PI3K inhibitor 3MA (10 mM). AKT phosphorylation on Ser473 was basally active in FTC133 cells, and it was activated under glucose-depleted conditions in WRO cells. 3MA effectively decreased AKT phosphorylation. Densitometry of the bands corresponding to PTEN (A) and of pAKT and AKT (B), normalised vs $\beta$ tubulin, is included. Data shown in this figure have been reproduced three times independently. 
re-suspension of the labelled cells (Fig. 1B). In WRO cells cultured in a glucose-containing standard medium, the uptake of 2-NBDG was negligible, while it increased upon incubation in a glucose-free medium (Fig. 1). The simplest explanation of this phenomenon is that 2-NBDG clearly competes with the glucose present in the culture medium. However, we noted that, in FTC133 cells, the basal uptake of 2-NBDG was much higher than in WRO cells, despite the presence of glucose in the culture medium, and it increased further when incubated in a glucose-free medium (Fig. 1).
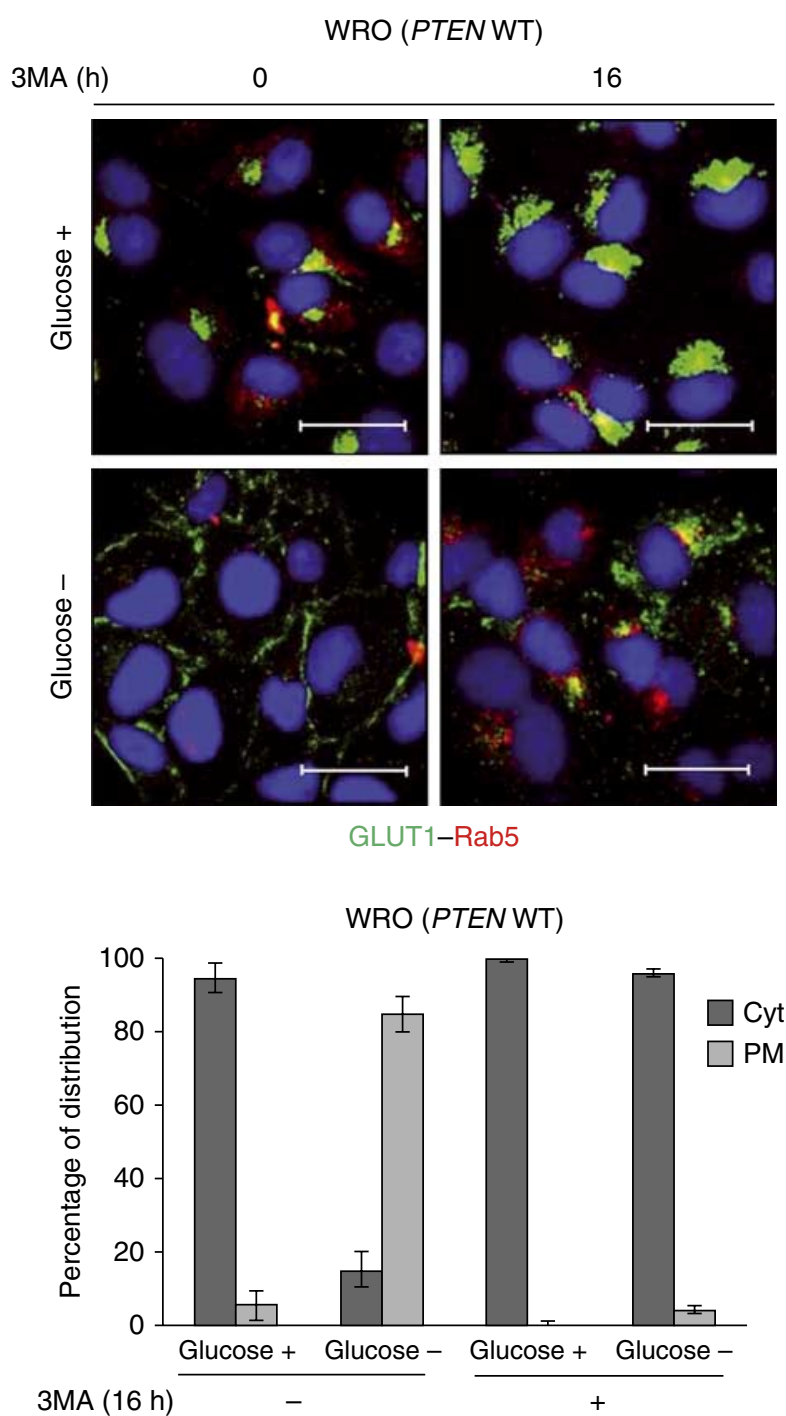

Figure 4

3-Methyladenine abrogates glucose starvation-induced plasma membrane (PM) translocation of GLUT1 in WRO cells. The cells adherent on coverslips were incubated in the presence or absence of 3MA for 16 in a glucosecontaining or glucose-free medium. At the end, the coverslips were processed for immunofluorescence staining of GLUT1 (in green) and Rab5 (in red; a marker of endocytic vesicles). Nuclei were stained with DAPI. Glucose starvation induced the PM relocation of GLUT1 in WRO cells.

\section{Glucose depletion differentially affects the membrane localisation of GLUT1 in WRO and FTC133 thyroid cancer cells}

Hypoxia triggers a metabolic switch towards aerobic glycolysis through HIF1 $\alpha$-mediated expression of GLUT proteins and glycolytic enzymes (Semenza 2012, Szablewski 2013). The metabolic stress induced by glucose depletion shares a common signalling pathway with hypoxia. The level of HIF1 $\alpha$ (HIF1A) expression was
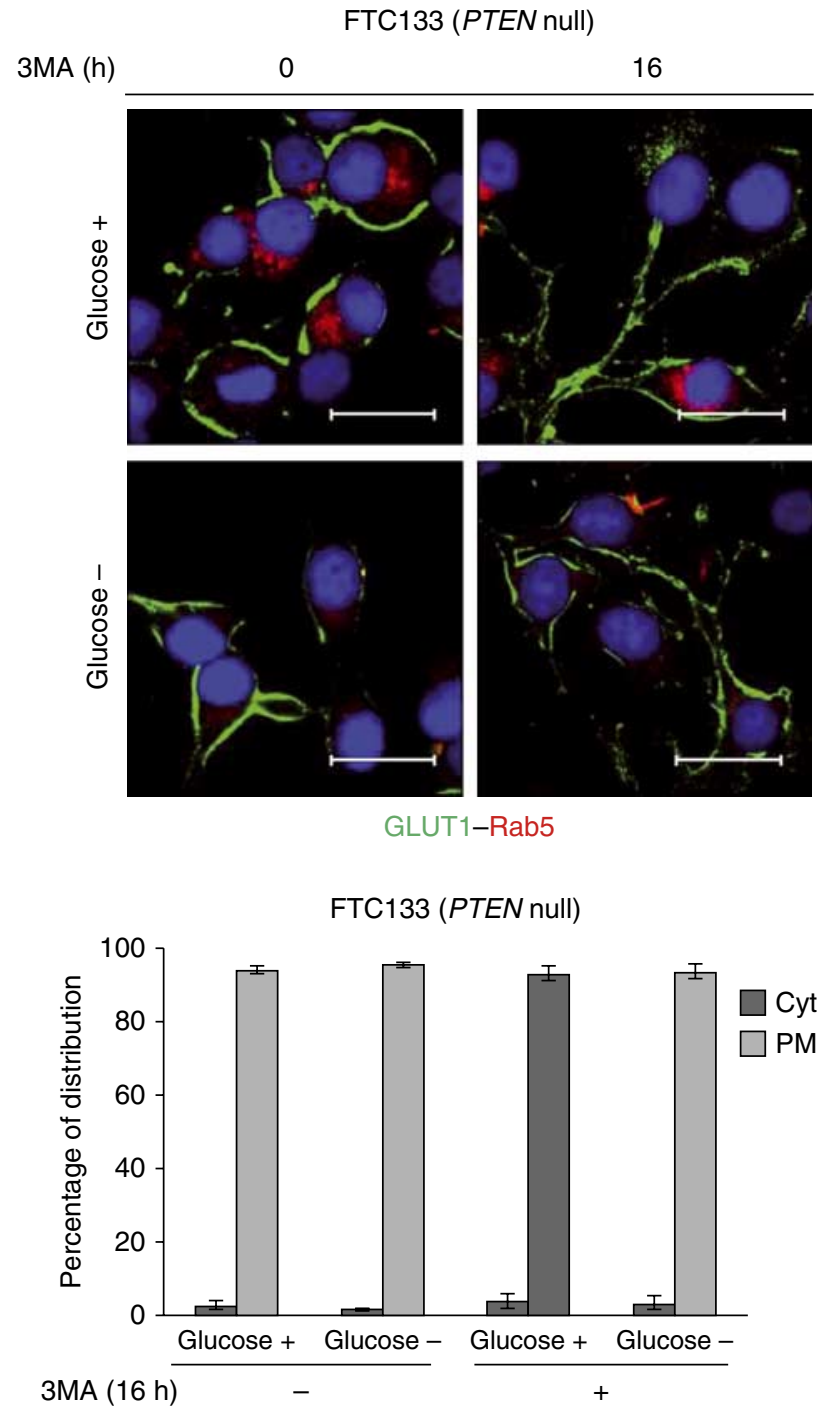

This effect was abrogated by 3MA. In FTC133 cells, GLUT1 was permanently localised to the PM, regardless of the presence or absence of glucose. Quantification of the relative distribution in cytoplasm (Cyt) vs PM of GLUT1 is included. Representative images and distribution of fluorescence (in percentage) of three independent experiments are shown. Full colour version of this figure available via http://dx.doi.org/10.1530/JME-14-0118. http://jme.endocrinology-journals.org DOI: 10.1530/JME-14-0118
(๑) 2014 Society for Endocrinology Printed in Great Britain 
higher in FTC133 cells than in WRO cells (Fig. 2A). Then, we looked at the expression of GLUT1, a GLUT that is abnormally over-expressed in highly aggressive and less differentiated thyroid cancers (Hosaka et al. 1992, Samih et al. 2000, Matsuzu et al. 2005). GLUT1 was expressed at a comparable level in WRO and FTC133 cells, and its level slightly increased upon incubation in a glucose-free medium (Fig. 2B). The above data suggested that membrane localisation rather than protein expression of GLUT1 could be the mechanistic cause for the differential uptake of glucose in the two cell lines. In the large majority $(>80 \%)$ of WRO cells cultured in a glucose-containing medium, GLUT1 was essentially localised to the cytoplasm, and it promptly translocated onto the PM on switching the culture to a glucose-free medium (Fig. 2C). Under glucose-depleted conditions, GLUT1 persisted on the PM of WRO cells for up to $48 \mathrm{~h}$ (not shown). On switching to a glucosedepleted medium, the membrane-associated GLUT1 fluorescence in WRO cells increased by approximately fourfold. By contrast, in FTC133 cells, GLUT1 was found permanently residing on the PM ( $>95 \%$ of GLUT1 fluorescence), regardless of whether the cells were cultured in the presence or the absence of glucose (Fig. 2C).

In the following experiments, we investigated the signalling pathway(s) involved in the glucose uptake and the membrane translocation of GLUT1 in response to glucose deprivation in the two thyroid cancer cell lines.
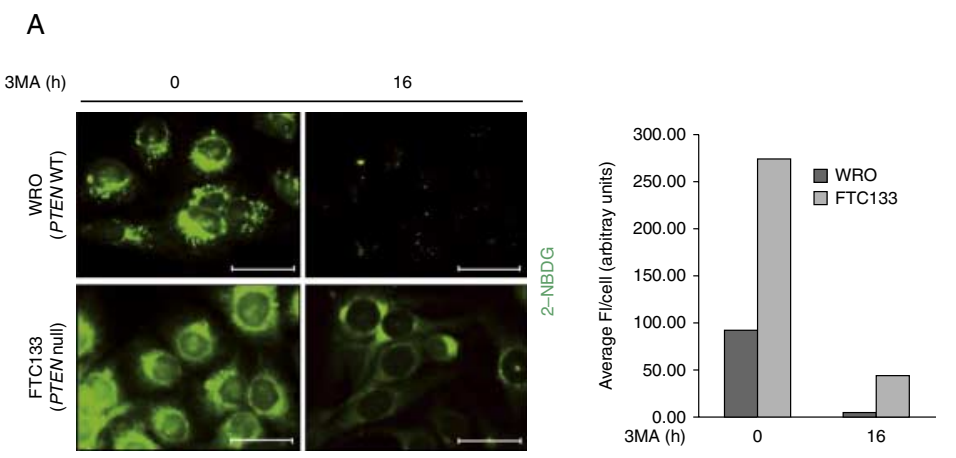

\section{PI3K drives the membrane translocation of GLUT1 in glucose-deprived WRO cells}

In FRTL-5 rat thyroid cells, the PM translocation of GLUT1 was shown to depend on the PI3K/AKT pathway (Samih et al. 2000). It has been reported that the activation of this pathway leads to increased HIF1 $\alpha$ levels (Zundel et al. 2000). Indeed, it is well documented that the PI3K-AKT pathway is abnormally activated in thyroid cancers (Saji \& Ringel 2010). Given that this pathway is controlled by the lipid kinase activity of PTEN, the above data could be explained with the altered expression of this oncosuppressor in the two cell lines. In fact, WRO cells express WT PTEN, whereas FTC133 cells are subjected to monoallelic deletion and bear an R130STOP mutant allele for PTEN (Weng et al. 2001, Saiselet et al. 2012). The latter leads to a truncated PTEN isoform that is not detectable in western blotting (Fig. 3A). The expression level of PTEN in WRO cells did not change after $24 \mathrm{~h}$ of incubation in a glucosefree medium (Fig. 3A). To determine as to what extent the AKT pathway was driving the membrane expression of GLUT1 in WRO and FTC133 thyroid cancer cells, we pharmacologically inhibited this pathway with 3MA, an inhibitor of PI3K. The PI3K-AKT pathway, as mirrored by Ser473 phosphorylation of AKT, was basally active (regardless of the presence or absence of glucose in the medium) in FTC133 cells, consistent with the lack of PTEN, and it was activated in WRO cells upon cultivation under glucose-free condition (Fig. 3B). In WRO cells, Ser473 phosphorylation of AKT was completely abrogated in the presence of $10 \mathrm{mM}$ 3MA (Fig. 3B). By contrast,

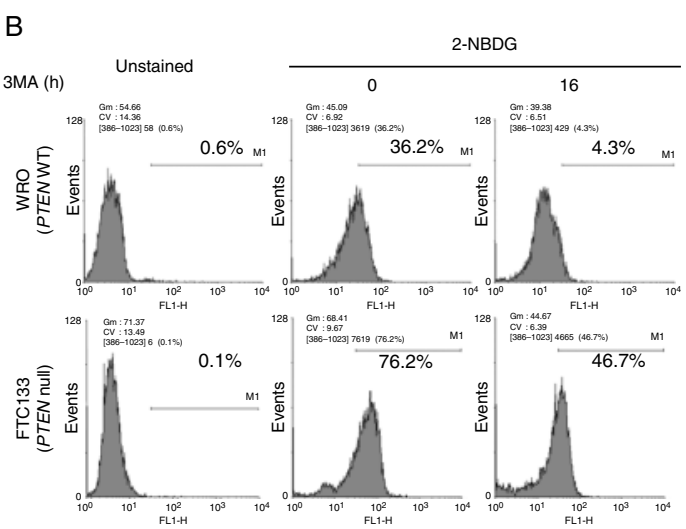

\section{Figure 5}

3MA differentially affects the uptake of glucose in WRO and FTC133 cells. (A) 2-NBDG uptake was monitored in both cell lines cultured for $16 \mathrm{~h}$ in a glucose-free medium in the absence or presence of 3MA. 3MA completely arrested the uptake of 2-NBDG in WRO cells under glucosedepleted conditions, while it had a milder effect in FTC133 cells. ImageJ quantification of 2-NBDG in the cells of representative fields is shown in the right panel. Scale bar $=20 \mu \mathrm{m}$. Magnification $=63 \times$. (B) Cytofluorometry assessment of 2-NBDG uptake in the cells cultured as above. Representative cytofluorograms are shown. This experiment further confirmed the inhibitory effect of 3MA on the uptake of glucose. Data shown in this figure have been reproduced four times independently. Full colour version of this figure available via http://dx.doi.org/10.1530/JME-14-0118. 
residual phospho-Ser473-AKT was still detectable in FTC133 cells even in the presence of 3MA, especially in glucose-depleted conditions (Fig. 3B). To be noted, the cellular level of total AKT was reduced in glucose-deprived cultures, especially in FTC133 cells.

We then looked at the phenotypic consequences of the activation and inhibition of the AKT pathway. 3MA had no effect on the membrane expression of GLUT1 in FTC133 cells, whereas, in WRO cells incubated in a glucose-free medium, it strongly limited the membrane translocation of GLUT1, which accumulated in a paraGolgian area (Fig. 4). This observation was confirmed by image quantification of the expression of GLUT1 in the Cyt and PM compartments (Fig. 4, lower panels).

\section{Inhibition of the PI3K-AKT pathway differentially affects the glucose uptake in WRO and FTC133 cells}

Then, we assessed the effects of 3MA on the uptake efficiency of glucose in WRO and FTC133 cells. The cells were plated on coverslips and incubated with the 2 -NBDG probe in the absence or presence of 3MA in a glucose-free medium. At the end, the cells were imaged under the microscope and cell-associated fluorescence was quantified using the ImageJ Software. 3MA completely abrogated the uptake of 2-NBDG in WRO cells, while it had a milder effect in FTC133 cells (Fig. 5A). Parallel cultures in Petri dishes were used for cytofluorometric quantification of fluorescence-positive cells. Cytofluorometry data indicated that a large
A

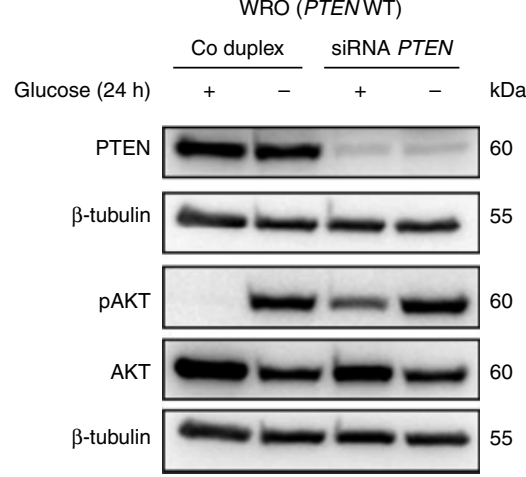

B
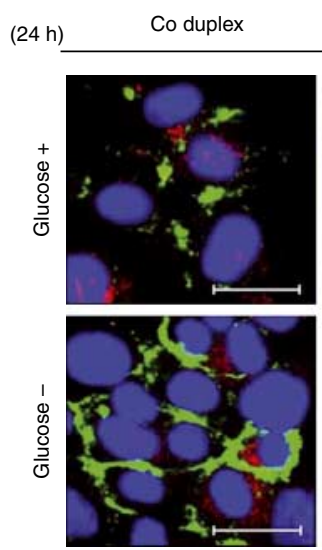

WRO (PTENWT)

SIRNA PTEN

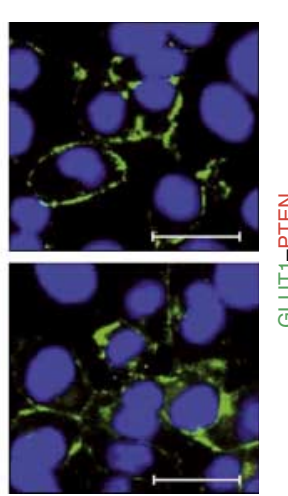

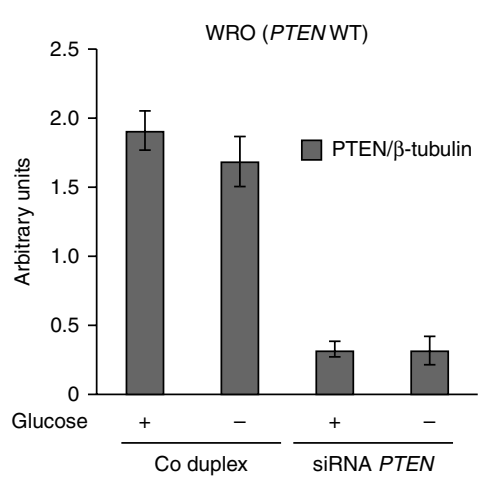
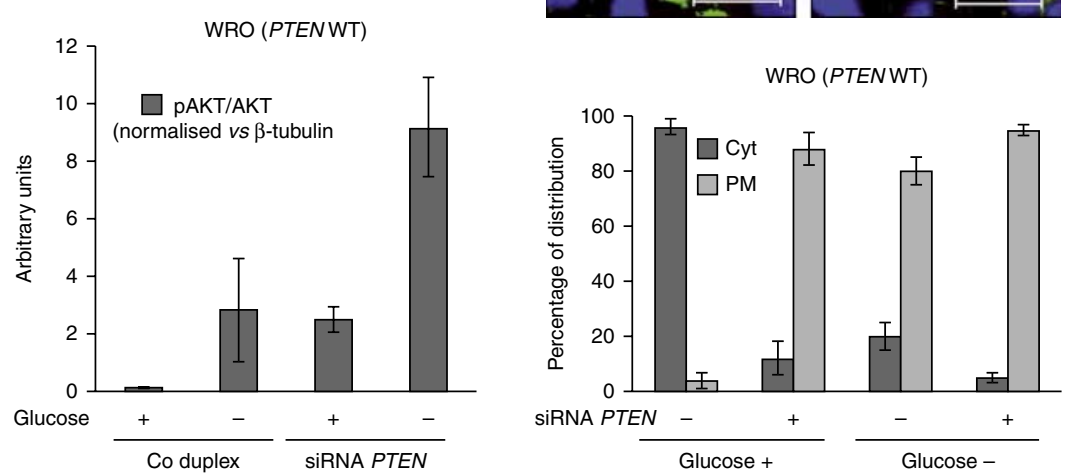

Figure 6

Silencing of PTEN in WRO cells determines the membrane translocation of GLUT1. (A) Knockdown of PTEN mRNA efficiently reduced ( $>80 \%$ ) the expression of the PTEN protein in WRO cells (data obtained with one of the two siRNA oligonucleotides targeting two different exons of PTEN are shown). The activation status of the AKT pathway was assessed by western blotting after siRNA silencing of PTEN and $24 \mathrm{~h}$ of incubation in a glucosecontaining or glucose-free medium. Basal phosphorylation of AKT increased upon silencing PTEN and further increased under glucose starvation. Densitometry quantification of the AKT activation status $(n=3)$ is reported in the lower panels (pAKT/AKT was normalised vs $\beta$-tubulin).
(B) Subcellular expression of GLUT1 (in green) and PTEN (in red) after siRNA silencing of PTEN and $24 \mathrm{~h}$ of incubation in a glucose-containing or glucose-free medium. Nuclei are stained with DAPI. Down-regulation of PTEN promoted the cytoplasmic (Cyt) to plasma membrane (PM) relocation of GLUT1. ImageJ quantification of this phenomenon is included. Note that $>80 \%$ of the PTEN siRNA-transfected cells were negative for PTEN staining. Scale bar $=20 \mu \mathrm{m}$. Magnification $=63 \times$. Representative images and distribution of fluorescence (in percentage) of three independent experiments are shown. Full colour version of this figure available via http://dx.doi.org/ 10.1530/JME-14-0118. http://jme.endocrinology-journals.org DOI: 10.1530/JME-14-0118
(C) 2014 Society for Endocrinology Printed in Great Britain 
proportion of FTC133 cells could take up glucose despite the presence of 3MA (Fig. 5B).

\section{Genetic manipulations of the PTEN expression and trafficking of GLUT1}

The above findings are consistent with the involvement of the PI3K/AKT pathway in the trafficking of GLUT1positive vesicles in both WRO and FTC133 cancer cells.

To determine whether PTEN is involved in the trafficking of GLUT1 in thyroid cancer cells, we genetically manipulated the expression of PTEN in WRO and FTC133 cells. First, we post-transcriptionally silenced PTEN in WRO cells. The transfection with a PTEN-specific siRNA elicited (on average) a $>80 \%$ down-regulation of the expression of PTEN protein (Fig. 6A). The genetic silencing

A
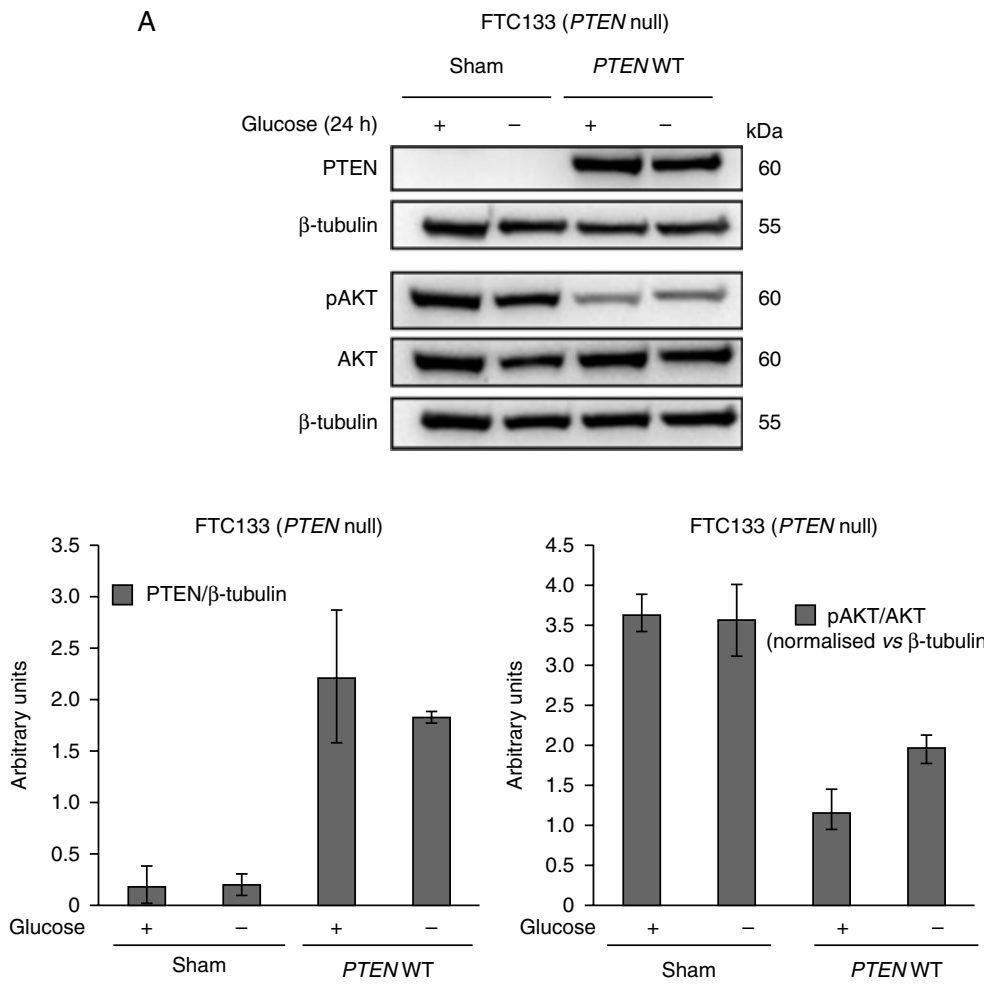

of PTEN expression was reflected on the activation of the AKT pathway. To be noted, under glucose-free condition, AKT was phosphorylated regardless of the expression level of PTEN (Fig. 6A, densitometry in lower panels). Parallel cultures were set on coverslips for immunofluorescence staining of GLUT1 and PTEN. The latter was included to monitor the cells that had been effectively silenced for PTEN. As a result of PTEN silencing, in a large proportion of the cells, GLUT1 was found on the PM (Fig. 6B). As predictable, this effect was more evident in the culture incubated in a glucose-free medium (Fig. 6B). Quantification by ImageJ analysis confirmed the PM translocation of GLUT1 upon PTEN knockdown.

Then, we transgenically over-expressed PTEN in PTEN-deficient FTC133 cells (Fig. 7A). The ectopic
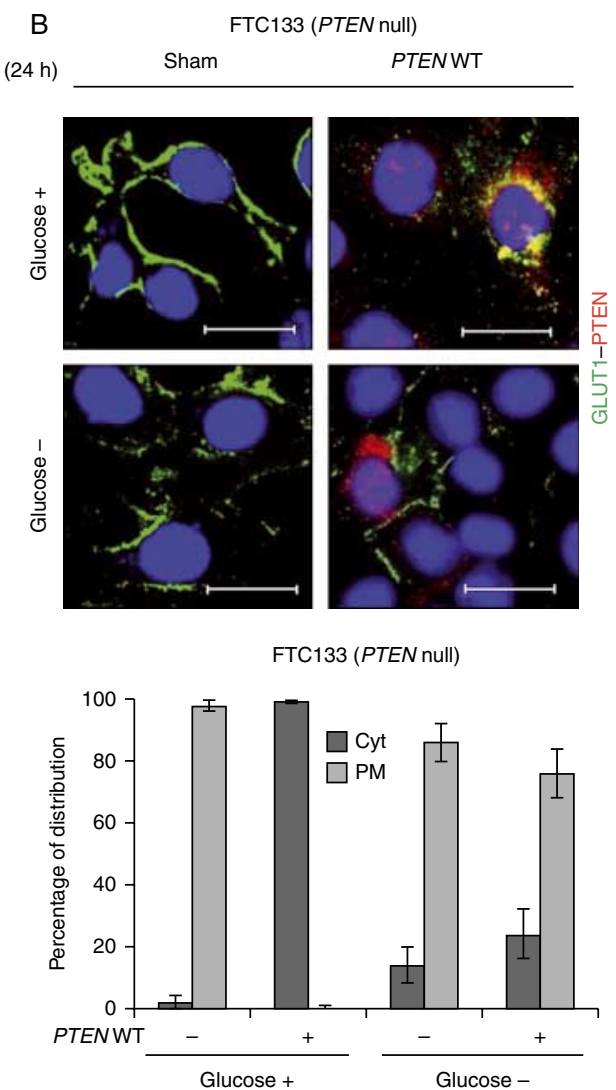

Figure 7

Transgenic expression of PTEN in FTC133 cells precludes the membrane translocation of GLUT1. (A) Transgenic expression of WT PTEN in FTC133 cells was controlled by western blotting assay. The blot was stripped and re-probed to assay the phosphorylation status of AKT. Data show that ectopic expression of PTEN greatly inhibited the phosphorylation of AKT independent of the presence or absence of glucose in the culture medium. Densitometric analysis $(n=3)$ of the AKT activation status is included (pAKT/AKT was normalised vs $\beta$-tubulin). (B) Subcellular expression of GLUT1 (in green) and PTEN (in red) in transfected FTC133 cells after $24 \mathrm{~h}$ of incubation in a glucose-containing or glucose-free medium. Nuclei are stained with DAPI. Under glucose-containing culture condition, GLUT1 appears clustered in a perinuclear-para-Golgian area and, in glucose-free culture condition, only a small amount of GLUT1 appears to reach the plasma membrane (PM). In PTEN-transfected cultures, $>60 \%$ of the cells were positive for PTEN immunofluorescence. Ectopic expression of PTEN largely precluded the PM relocation of GLUT1. ImageJ quantification of this phenomenon is included. Representative images and distribution of fluorescence (in percentage) of three independent experiments are shown. Cyt, cytoplasm. Scale bar $=20 \mu \mathrm{m}$. Magnification $=63 \times$. Full colour version of this figure available via http://dx.doi.org/10.1530/JME-14-0118.

Published by Bioscientifica Ltd 
expression of PTEN was paralleled by the inactivation $(\sim 75 \%)$ of the AKT pathway (see densitometry in lower panels), probably reflecting the de-phosphorylation of PIP3 in PIP2 operated by PTEN. To be noted, in PTENtransfected cells, AKT remained largely inactive even when cultured in a glucose-free medium (Fig. 7A). In a parallel experiment, the cells adherent on coverslip were stained for GLUT1 and PTEN. In the transfected cells (positive for PTEN), GLUT1 was mainly clustered in a perinuclear-para-Golgian region, though this effect was somehow mitigated when the cells were cultured in a glucose-free medium (Fig. 7B). Based on ImageJ quantification, ectopic expression of PTEN almost completely abrogated the PM-associated GLUT1 fluorescence translocation of GLUT1 in transfected FTC133 cells cultured in the presence of glucose (Fig. 7B).

To determine the functional consequences of the PTEN-dependent relocation of GLUT1 on glucose uptake, we measured the uptake of 2-NBDG in the transfected cell populations. The proportion of cells positive for 2-NBDG almost doubled in PTEN-silenced WRO cells compared with control duplex-transfected cells and almost halved in transfected cells expressing transgenic PTEN compared with the control Sham-transfected counterparts (Fig. 8).

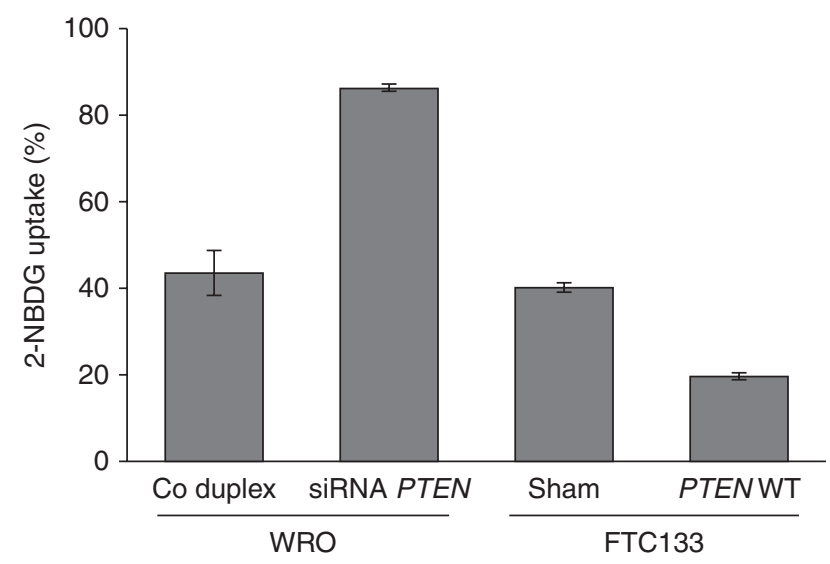

Figure 8

PTEN drives the glucose uptake in thyroid cancer cells. WRO and FTC133 cells adherent on Petri dishes were transfected with a PTEN-specific siRNA or with a plasmid harbouring the PTEN CDNA respectively. Sham-transfection was included as a control. The cells were incubated for the optimal time to attain the highest efficiency of endogenous PTEN knockdown or of ectopic PTEN expression in WRO and in FTC133 cells respectively. The cells were then loaded with the fluorescent 2-NBDG probe and analysed by cytofluorometry. The histogram shows the quantification of uptake (average \pm s.D.) of four experiments. Note that control duplextransfected WRO cells show an abnormally high uptake of 2-NBDG, probably due to the stress associated with Lipofectamine transfection. The data clearly indicate that PTEN is a master regulator of glucose uptake in WRO and in FTC133 cells.

\section{Discussion}

To sustain their high proliferative rate, cancer cells adapt their metabolism towards the aerobic glycolysis (Tong et al. 2009). Thyroid cancer cells also show a high rate of glycolysis (Andrade et al. 2012). Immunohistochemical studies have shown that enhanced glucose uptake in cancer cells correlates with over-expression of GLUTs, in most cases GLUT1 (Haber et al. 1997, Ciampi et al. 2008). Herein, we show that the oncosuppressor PTEN plays a dominant role in the membrane expression of GLUT1 and glucose uptake in thyroid cancer cells. It has been shown that the ectopic hyper-expression of PTEN contrasts the uptake and the large glycolytic consumption of glucose observed in proliferating cancer cells (Garcia-Cao et al. 2012). Consistently, we found that the transgenic reintroduction of PTEN in FTC133 abrogates the membrane expression of GLUT1. This effect was evident in glucosefed cells, while, in the glucose-deprived cells, the absence of glucose triggered anyhow the membrane translocation of GLUT1 (Fig. 7B). Thus, the absence of glucose is 'dominant' in driving GLUT1 membrane translocation, regardless of the presence of PTEN as in fact occurs in WRO cells as well (Figs 2C, 4 and 6B). Glucose depletion is known to activate the AMPK pathway, which is also involved in the membrane expression of GLUT1 (Andrade et al. 2012).

Conversely, we show that the lack of PTEN expression has a dominant effect on the membrane expression of GLUT1 independent of the level of glucose available. Notably, in PTEN-expressing cancer cells, the scarce availability of glucose favoured the expression of GLUT1 on the PM. The PTEN-dependent relocation of GLUT1 had functional consequences in terms of glucose uptake, as the silencing of PTEN in WRO cells greatly increased, and conversely the ectopic expression of PTEN in FTC133 cells greatly reduced, the uptake rate of 2-NBDG. These facts have relevance in terms of imaging diagnosis. Imaging by FDG-PET has the potential to reveal unsuspected tumours, including thyroid tumours, commonly indicated as 'incidentaloma' because of their accidental discovery (Bogsrud et al. 2010, Hsiao et al. 2011, Pagano et al. 2011, Bertagna et al. 2012). Novel non-radioactive methods for imaging the glucose internalisation by cancer cells are being developed (Walker-Samuel et al. 2013), underscoring the importance of glucose uptake as a diagnostic readout of cancer proliferation.

The uptake of glucose in thyroid cancer cells has been shown to be inversely correlated with the uptake of iodide (the so-called 'flip-flop' phenomenon), a feature

Published by Bioscientifica Ltd. 
of highly aggressive thyroid tumours that are associated with increased lethality (Filetti et al. 1986, Frilling et al. 2001, Bläser et al. 2006, Grabellus et al. 2012). In addition, a link between the expressions of GLUT molecules and the pGp responsible for chemoresistance has been shown (Seo et al. 2009). Therefore, understanding the signalling pathways that govern the membrane expression of GLUTs and the glucose uptake in thyroid cancer cells also has prognostic and therapeutic implications.

\section{Declaration of interest}

The authors declare that there is no conflict of interest that could be perceived as prejudicing the impartiality of the research reported.

\section{Funding}

F M was supported by a PhD fellowship granted by the 'Associazione Franca Capurro per Novara - Onlus'. S P is recipient of a PhD fellowship in Biotechnology for Human Health. C F was supported by a 'Liberato Di Lauro' post-doc fellowship granted by the 'Associazione per la Ricerca Medica Ippocrate-Rhazi' (Novara, Italy). R T is recipient of a 'Progetto Lagrange' PhD student fellowship (Cassa di Risparmio di Torino, Italy). The WRO and FTC-133 thyroid cancer cell lines were kindly provided by Dr Francesco Frasca (University of Catania, Italy). Research in the laboratory of C I was supported by Comoli, Ferrari \& SpA (Novara, Italy).

\section{References}

Andrade BM, Cazarin J, Zancan P \& Carvalho DP 2012 AMP-activated protein kinase upregulates glucose uptake in thyroid PCCL3 cells independent of thyrotropin. Thyroid 22 1063-1068. (doi:10.1089/thy. 2012.0041)

Bertagna F, Treglia G, Piccardo A \& Giubbini R 2012 Diagnostic and clinical significance of F-18-FDG-PET/CT thyroid incidentalomas. Journal of Clinical Endocrinology and Metabolism 97 3866-3875. (doi:10.1210/ jc.2012-2390)

Bläser D, Maschauer S, Kuwert T \& Prante O 2006 In vitro studies on the signal transduction of thyroidal uptake of ${ }^{18}$ F-FDG and ${ }^{131}$ I-Iodide. Journal of Nuclear Medicine 47 1382-1388.

Boellaard R, O'Doherty MJ, Weber WA, Mottaghy FM, Lonsdale MN, Stroobants SG, Oyen WJ, Kotzerke J, Hoekstra OS, Pruim J et al. 2010 FDG PET and PET/CT: EANM procedure guidelines for tumour PET imaging: version 1.0. European Journal of Nuclear Medicine and Molecular Imaging 37 181-200. (doi:10.1007/s00259-009-1297-4)

Bogsrud TV, Karantanis D, Nathan MA, Mullan BP, Wiseman GA, Kasperbauer JL, Reading CC, Björo T, Hay ID \& Lowe VJ 2010 The prognostic value of 2-deoxy-2-[ $\left.{ }^{18} \mathrm{~F}\right]$ fluoro-D-glucose positron emission tomography in patients with suspected residual or recurrent medullary thyroid carcinoma. Molecular Imaging and Biology 12 547-553. (doi:10.1007/s11307-009-0276-2)

Busk M, Horsman MR, Kristjansen PE, van der Kogel AJ, Bussink J \& Overgaard J 2008 Aerobic glycolysis in cancers: implications for the usability of oxygen-responsive genes and fluorodeoxyglucose-PET as markers of tissue hypoxia. International Journal of Cancer 122 2726-2734. (doi:10.1002/ijc.23449)

Castino R, Bellio N, Nicotra G, Follo C, Trincheri NF \& Isidoro C 2007 Cathepsin D-Bax death pathway in oxidative stressed neuroblastoma cells. Free Radical Biology \& Medicine 42 1305-1316. (doi:10.1016/ j.freeradbiomed.2006.12.030)
Ciampi R, Vivaldi A, Romei C, Del Guerra A, Salvadori P, Cosci B, Pinchera A \& Elisei R 2008 Expression analysis of facilitative glucose transporters (GLUTs) in human thyroid carcinoma cell lines and primary tumors. Molecular and Cellular Endocrinology 291 57-62. (doi:10.1016/j.mce. 2008.05.003)

Filetti S, Vetri M, Damante G \& Belfiore A 1986 Thyroid autoregulation: effect of iodine on glucose transport in cultured thyroid cells. Endocrinology 118 1395-1400. (doi:10.1210/endo-118-4-1395)

Frilling A, Tecklenborg K, Görges R, Weber F, Clausen M \& Broelsch EC 2001 Preoperative diagnostic value of [(18)F] fluorodeoxyglucose positron emission tomography in patients with radioiodine-negative recurrent well-differentiated thyroid carcinoma. Annals of Surgery 234 804-811. (doi:10.1097/00000658-200112000-00012)

Garcia-Cao I, Song MS, Hobbs RM, Laurent G, Giorgi C, de Boer VC, Anastasiou D, Ito K, Sasaki AT, Rameh L et al. 2012 Systemic elevation of PTEN induces a tumor-suppressive metabolic state. Cell 149 49-62. (doi:10.1016/j.cell.2012.02.030)

Grabellus F, Nagarajah J, Bockisch A, Schmid KW \& Sheu SY 2012 Glucose transporter 1 expression, tumor proliferation, and iodine/glucose uptake in thyroid cancer with emphasis on poorly differentiated thyroid carcinoma. Clinical Nuclear Medicine 37 121-127. (doi:10.1097/ RLU.0b013e3182393599)

Haber RS, Weiser KR, Pritsker A, Reder I \& Burstein DE 1997 GLUT1 glucose transporter expression in benign and malignant thyroid nodules. Thyroid 7 363-367. (doi:10.1089/thy.1997.7.363)

Hosaka Y, Tawata M, Kurihara A, Ohtaka M, Endo T \& Onaya T 1992 The regulation of two distinct glucose transporter (GLUT1 and GLUT4) gene expressions in cultured rat thyroid cells by thyrotropin. Endocrinology 131 159-165. (doi:10.1210/endo.131.1.1319316)

Hsiao YC, Wu PS, Chiu NT, Yao WJ, Lee BF \& Peng SL 2011 The use of dual-phase ${ }^{18}$ F-FDG PET in characterizing thyroid incidentalomas. Clinical Radiology 66 1197-1202. (doi:10.1016/j.crad.2011.08.005)

Koppenol WH, Bounds PL \& Dang CV 2011 Otto Warburg's contributions to current concepts of cancer metabolism. Nature Reviews. Cancer 11 325-337. (doi:10.1038/nrc3038)

Matsuzu K, Segade F, Wong M, Clark OH, Perrier ND \& Bowden DW 2005 Glucose transporters in the thyroid. Thyroid 15 545-550. (doi:10.1089/ thy.2005.15.545)

Morani F, Pagano L, Prodam F, Aimaretti G \& Isidoro C 2012 Loss of expression of the oncosuppressor PTEN in thyroid incidentalomas associates with GLUT1 plasmamembrane expression. Panminerva Medica 54 59-63.

Newsholme EA, Crabtree B \& Ardawi MS 1985 The role of high rates of glycolysis and glutamine utilization in rapidly dividing cells. Bioscience Reports 5 393-400. (doi:10.1007/BF01116556)

O'Neil RG, Wu L \& Mullani N 2005 Uptake of a fluorescent deoxyglucose analog (2-NBDG) in tumor cells. Molecular Imaging and Biology $\mathbf{7}$ 388-392. (doi:10.1007/s11307-005-0011-6)

Pagano L, Samà MT, Morani F, Prodam F, Rudoni M, Boldorini R, Valente G, Marzullo P, Baldelli R, Appetecchia M et al. 2011 Thyroid incidentaloma identified by ${ }^{18} \mathrm{~F}$-fluorodeoxyglucose positron emission tomography with CT (FDG-PET/CT): clinical and pathological relevance. Clinical Endocrinology 75 528-534. (doi:10.1111/j.1365-2265.2011. 04107.x)

Saiselet M, Floor S, Tarabichi M, Dom G, Hébrant A, van Staveren WC \& Maenhaut C 2012 Thyroid cancer cell lines: an overview. Frontiers in Endocrinology 3 133. (doi:10.3389/fendo.2012.00133)

Saji M \& Ringel MD 2010 The PI3K-Akt-mTOR pathway in initiation and progression of thyroid tumors. Molecular and Cellular Endocrinology 321 20-28. (doi:10.1016/j.mce.2009.10.016)

Samih N, Hovsepian S, Aouani A, Lombardo D \& Fayet G 2000 Glut-1 translocation in FRTL-5 thyroid cells: role of phosphatidylinositol 3-kinase and N-glycosylation. Endocrinology 141 4146-4155. (doi:10.1210/endo.141.11.7793)

Semenza GL 2012 Hypoxia-inducible factors in physiology and medicine. Cell 148 399-408. (doi:10.1016/j.cell.2012.01.021) 
Seo S, Hatano E, Higashi T, Nakajima A, Nakamoto Y, Tada M, Tamaki N, Iwaisako K, Kitamura K, Ikai I et al. 2009 P-glycoprotein expression affects ${ }^{18} \mathrm{~F}$-fluorodeoxyglucose accumulation in hepatocellular carcinoma in vivo and in vitro. International Journal of Oncology 34 1303-1312. (doi:10.3892/ijo_00000257)

Szablewski L 2013 Expression of glucose transporters in cancers. Biochimica et Biophysica Acta 1835 164-169. (doi:10.1016/j.bbcan. 2012.12.004)

Tong X, Zhao F, Mancuso A, Gruber JJ \& Thompson CB 2009 The glucose-responsive transcription factor ChREBP contributes to glucose-dependent anabolic synthesis and cell proliferation. PNAS 106 21660-21665. (doi:10.1073/pnas.0911316106)

Trincheri NF, Nicotra G, Follo C, Castino R \& Isidoro C 2007 Resveratrol induces cell death in colorectal cancer cells by a novel pathway involving lysosomal cathepsin D. Carcinogenesis 28 922-931. (doi:10.1093/carcin/bgl223)

Walker-Samuel S, Ramasawmy R, Torrealdea F, Rega M, Rajkumar V, Johnson SP, Richardson S, Gonçalves M, Parkes HG, Arstad E et al. 2013 In vivo imaging of glucose uptake and metabolism in tumors. Nature Medicine 19 1067-1072. (doi:10.1038/nm.3252)

Weng LP, Gimm O, Kum JB, Smith WM, Zhou XP, Wynford-Thomas D, Leone G \& Eng C 2001 Transient ectopic expression of PTEN in thyroid cancer cell lines induces cell cycle arrest and cell type-dependent cell death. Human Molecular Genetics 10 251-258. (doi:10.1093/hmg/10.3.251)

Zundel W, Schindler C, Haas-Kogan D, Koong A, Kaper F, Chen E, Gottschalk AR, Ryan HE, Johnson RS, Jefferson AB et al. 2000 Loss of PTEN facilitates HIF-1-mediated gene expression. Genes and Development 14 391-396. (doi:10.1101/gad.14.4.391)

Received in final form 1 August 2014

Accepted 14 August 2014

Accepted Preprint published online 14 August 2014
() 2014 Society for Endocrinology Printed in Great Britain
Published by Bioscientifica Ltd. 